Revista Calidad en la Educación Superior

Programa de Autoevaluación Académica

Universidad Estatal a Distancia, Costa Rica

ISSN 1659-4703

revistacalidad@uned.ac.cr

\title{
CARACTERÍSTICAS DE LA RETROALIMENTACIÓN COMO PARTE DE LA ESTRATEGIA EVALUATIVA DURANTE EL PROCESO DE ENSEÑANZA APRENDIZAJE EN ENTORNOS VIRTUALES: UNA PERSPECTIVA TEÓRICA
}

\author{
CHARACTERISTICS OF FEEDBACK AS PART OF THE EVALUATIVE STRATEGY \\ DURING THE TEACHING AND LEARNING PROCESS IN VIRTUAL \\ ENVIRONMENTS: A THEORETICAL PERSPECTIVE
}

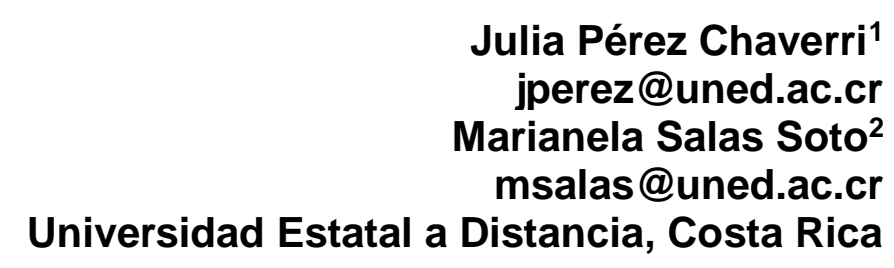

\author{
Volumen 7, Número 1 \\ Mayo 2016 \\ pp. $175-204$
}

Recibido: 30 de agosto, 2015

Aprobado: 12 de abril, 2016

\footnotetext{
${ }^{1}$ Magister en educación con énfasis en la enseñanza del español S.L. Magister en educación con énfasis en docencia universitaria, profesora de educación especial profesora de formación docente, Centro de Capacitación en Educación a Distancia en la UNED, diseñadora y facilitadora de cursos y talleres virtuales.

2 Doctora en educación con énfasis en calidad del e-learning. Magister en planificación curricular, profesora de formación docente, Centro de Capacitación en Educación a Distancia en la UNED, diseñadora y facilitadora de cursos y talleres virtuales.
} 


\section{Resumen}

Esta revisión bibliográfica se llevó a cabo para identificar las condiciones y características de la retroalimentación como parte de la estrategia evaluativa durante el proceso de enseñanza aprendizaje en entornos virtuales. Estos fundamentos permiten orientar el quehacer de la docencia que se lleva a cabo en la Universidad Estatal a Distancia (UNED). El análisis de este estudio se basó en una revisión de literatura de los autores más reconocidos y actuales en el campo de la evaluación de los aprendizajes y su propósito fue recuperar la mayor cantidad posible de aportes sobre el papel de la retroalimentación y cómo esta puede potenciar procesos de aprendizaje más profundos y significativos. Los hallazgos más importantes se centran en la retroalimentación como proceso dialógico y colaborativo en el marco de la evaluación alternativa: formativa y formadora que se ve potenciada por las posibilidades de apertura, personalización, interactividad, ubicuidad, inmediatez, flexibilidad, interoperabilidad y colaboración que ofrecen los entornos virtuales de aprendizaje.

Palabras claves: retroalimentación; feedback; estrategia de evaluación; proceso de enseñanza y aprendizaje; entornos virtuales de aprendizaje.

\section{Summary}

This bibliographical review was conducted to identify the conditions and characteristics of feedback as part of the evaluation strategy during the teaching-learning process in virtual environments. These fundamentals orient the work of the teaching that takes place at the Universidad Estatal a Distancia.

The analysis of this study is based on a literature review of the most recognized and present authors in the field of learning assessment, and its purpose was to recover the maximum amount of contributions on the role of feedback, and how it can enhance deeper and more meaningful learning processes.

The most important findings focus on the feedback as a dialogical and collaborative process within the framework of alternative assessment: formative and forming, that is enhanced by opening possibilities, personalization, interactivity, ubiquity, immediacy, flexibility, interoperability and collaboration offering virtual learning environments.

Keywords: feedback; evaluative strategy; teaching and learning process; virtual learning environments. 


\section{El problema y su justificación}

La incorporación de las plataformas virtuales y la creación del Modelo Pedagógico en el 2004, permitieron que la Universidad Estatal a Distancia (UNED) repensara la dirección de los procesos de enseñanza y aprendizaje. Es así que en dicho documento se plantea que:

1. Los procesos educativos deben estar centrados en el estudiante, por lo que este debe ser un participante activo de su propio proceso de aprendizaje (p. 28).

2. Los equipos docentes deben trabajar de manera coordinada para favorecer la autonomía, la autorregulación, la autoevaluación y la coevaluación en el estudiante (p. 33).

3. Las diferentes tecnologías de la información y comunicación, deben apoyar la academia, especialmente cuando se refiere a favorecer los procesos de interacción y aprendizaje de estudiantes, con y entre estudiantes (p. 24).

Los aspectos mencionados anteriormente, plantean nuevos retos administrativos, tecnológicos, académicos y docentes, puesto que ya no se trata de ofertar asignaturas con una dinámica que incluye "entrega de dos tareas y realización de dos exámenes", como tradicionalmente se hizo por muchos años. Precisamente, para subsanar ciertos vacíos de nuestra modalidad a distancia, es que se impulsa el uso pedagógico de plataformas virtuales de aprendizajes. Así que para el 2010, en el documento Cómo ofertar y diseñar cursos en línea (2010), establece una serie de condiciones que deben tomarse en cuenta para que la plataforma se transforme en una verdadera aula virtual. Algunas de las condiciones que se deben asumir son:

1. Utilizar foros de consulta.

2. Digitalizar materiales de lectura.

3. Diseñar estrategias por medio de foros de discusión.

4. Elaborar instrumentos de evaluación propios para estas dinámicas.

5. Utilizar actividades que favorezcan el trabajo colaborativo. 
6. Atender las consultas de los estudiantes al menos cada dos días, tanto las que se envían por el correo de la plataforma, como las colocadas en el foro respectivo.

7. Moderar pedagógicamente dos o tres foros.

8. Usar las herramientas de evaluación que ofrece la plataforma.

9. Potenciar el desarrollo de la metacognición en los estudiantes.

10. Evaluar los resultados de los estudiantes y del curso en la plataforma (p. 31).

Entonces, a pesar de los esfuerzos que se han realizado en la UNED, todavía existen muchos vacíos sobre cómo implementar procesos de evaluación que favorezcan el aprendizaje centrado en el estudiante y cómo favorecer el aprendizaje a través de la evaluación. Lo anterior genera la necesidad de repensar qué es y cómo deben llevarse a cabo los procesos de realimentación como parte de la estrategia evaluativa en los entornos virtuales institucionales y la función que debe asumir la persona en su rol del evaluador desde lo planteado en el Modelo Pedagógico y en el Plan Académico vigentes.

Además, las universidades públicas y privadas, cada vez más están asumiendo el reto de incorporar la virtualidad y diferentes recursos tecnológicos; en ese sentido, implican que los roles docente y estudiante se tornan más complejos, demandan de ellos no solo nuevas responsabilidades en el proceso enseñanza-aprendizaje, sino también de manera más específica en el de evaluación.

Por lo tanto, esta investigación se vuelve fundamental pues permite recopilar fundamentos teóricos recientes sobre el término realimentación como parte de una propuesta evaluativa en el marco de un proceso educativo o formativo. En ese sentido, será preciso identificar cuáles son las características de este proceso, que repercute directamente en el desempeño del profesor y del estudiante, sobre todo cuando el acto educativo/formativo se lleva a cabo en un entorno virtual. 


\section{Marco teórico}

\subsection{De la evaluación a la retroalimentación}

Por muchas décadas medir se convirtió en la acción central de la evaluación. Según Luzardo (s.a), este proceso era entendido como un proceso de cuantificación en el que se compara un objeto o una cualidad de ese objetivo a un parámetro previamente establecido (p. 77). Arancibia (1997) indica que cuando se habla de evaluación en un sistema educativo y más aún si se trata de un proceso de enseñanza y aprendizaje, es frecuente pensarlo desde un enfoque tradicional (p. 7). Es urgente tomar en cuenta que cuando se habla de evaluación de los aprendizajes, se habla de resultados, pero también de procesos.

A criterio de Arancibia (1997), cuando se lleva a cabo solo un proceso de medición, puede tener más efectos negativos que positivos en el proceso educativo. $\mathrm{Si}$, por el contrario, se complementa con un sistema de retroalimentación, puede tener un efecto directo y positivo en el aprendizaje de los estudiantes (p. 33). Por ende, cambiar de una evaluación tradicional a una evaluación que promueva la retroalimentación como parte del aprendizaje y el desarrollo integral de los estudiantes, requiere igualmente una trasformación del acto educativo. Precisamente Coll, Mauri y Rochera (2012) consideran que la evaluación es una práctica de actividad continua, formativa, reguladora, auténtica, participada y social. La evaluación constituye un sistema integrado en -y alineado o concordante con- las actividades de enseñanza y aprendizaje (p. 53).

De acuerdo a Barberá (2006), "la evaluación no es solamente evaluación del aprendizaje sino que es también evaluación para el aprendizaje" (p. 6). Entonces, desde una perspectiva más innovadora, la evaluación de los aprendizajes se puede concebir como un proceso: 
1. Para obtener informaciones sobre los aprendizajes que alcanza la persona, durante el proceso educativo. Con esta base, se toman decisiones para redireccionar el quehacer docente (Ministerio de Educación de Guatemala, 2010).

2. Para valorar cambios y resultados producidos en las personas que aprenden como consecuencia del proceso de enseñanza-aprendizaje (Fernández, 2005)

3. De modificación de actitud y visión, puesto que evaluar condiciona tanto al profesor como al estudiante para lograr aprendizajes de diferente nivel y profundidad (Sans Martín, 2008).

En esa línea, Fernández, García y González (2004) hacen referencia que un proceso de evaluación de los aprendizajes debe llevarse a cabo durante todo el acto educativo, ya que su objetivo es aportar retroalimentación y para orientar al aprendizaje (p. 2). Indican Bordas y Cabrera (2001) que la evaluación, más que continua, debe ser continuada:

Implica el concepto de "permanente" en el espacio y en el tiempo, en sentido horizontal y vertical. Por consiguiente, no afecta solo en situación directa de aprendizaje (aprendizaje formal) sino a toda clase de situaciones, formas y contextos; no afecta solo en aprendizajes que se realizan en determinados momentos sino a lo largo de toda la vida. Y siempre haciendo hincapié en la actitud de feedback permanente (p. 6).

En opinión de Durán y Valerio (2008), si el proceso de evaluación se enfoca desde lo alternativo permite que la retroalimentación constante fortalezca áreas débiles y potencie las áreas fuertes que posee el estudiante. Si se le comunica a tiempo al estudiante qué nivel de calidad presenta su producción o aprendizaje, puede servir de parámetro de comparación para que otros estudiantes lo usen como punto de referencia y como una estrategia para el intercambio de criterios y, de ese modo, fomentar retroalimentación. Según Jiménez (2015): 
el feedback deber ser descriptivo, simple y objetivamente claro y centrado sobre la actividad o tarea concreta, en cuyo caso disminuye la carga emocional del estudiantado dado que se representa la situación como manejable. Asimismo, puede darse de forma escrita u oral. Por el contrario, si el problema es planteado como una apreciación del docente o la docente acerca de la persona de la alumna o del alumno es más probable que se dé una reacción negativa o defensiva del alumnado, lo que conduce a que se pierda el propósito educacional del feedback y se altere la relación docente y personas en proceso de formación. (p. 8)

Por lo tanto, retroalimentación como parte de la estrategia evaluativa, se puede definir de la siguiente manera:

1. De acuerdo a Amaranti (2010), es una actividad dialógica en la que los profesores y estudiantes analizan los resultados de las evaluaciones en comparación con los criterios propuestos y se toman acuerdos de acciones que se pueden desarrollar para mejorar en pos de las metas de aprendizaje (p. 4).

2. Maldonado (2009) cita a Lara Sierra, quién define retroalimentación como "el retorno de información sobre el resultado de una actividad o un proceso" (p. 5).

3. Lozano y Tamez (2014) citan a Hattie y Timperley, quienes definen la retroalimentación como "La retroalimentación es la información que provee un agente como podría ser: un profesor, un compañero de equipo, un libro, uno mismo, sobre el desempeño académico de una actividad de aprendizaje" ( $p$. 200).

4. Según Amaranti Pece (2010), una acción crucial para transformar la evaluación en una oportunidad para aprender. La forma en que se van comunicando los resultados de una evaluación y las posibles acciones que se proponen al estudiante para mejorar (...) y aprender mediante la evaluación (p. 4). 
Maldonado (2009) explica que la retroalimentación o feedback integrada a la evaluación requiere una devolución de información procesada "para consolidar los conocimientos adquiridos y poder ir más allá en el proceso formativo" (p. 6). Termina, cuando el estudiante ha logrado el objetivo de aprendizaje propuesto en la evaluación. Para que la retroalimentación sea parte de la evaluación, todos los involucrados en el proceso educativo o formativo, deben tener claro cuáles son los aprendizajes propuestos antes de iniciar tal proceso, de esa manera, el estudiante puede ir generando las evidencias de ese aprendizaje. Para que estas evidencias sean entregas progresivas, debe darse un proceso de comunicación adecuado y esto va más allá de la simple transmisión de información (Ávila, 2009).

\subsection{Aprendizaje y retroalimentación}

El concepto de aprendizaje no es el objeto de estudio de este trabajo; pero si el aprendizaje es el eje y propósito de todo cuanto se persigue cuando se brinda una adecuada retroalimentación de los procesos educativos. Por lo tanto, nos referimos brevemente al término de aprendizaje el cual proviene de latin prehéndere que quiere decir coger atrapar, lo cual nos refiere a una acción de adquisición, de apoderarse de algo, para llegar a denotar la adquisición de conocimiento de alguna cosa por medio del estudio o experiencia.

En el sentido más amplio, el aprendizaje ocurre, según Woolfolk (2012) cuando la experiencia (incluyendo la práctica) genera un cambio relativamente permanente en los conocimientos o las conductas de un individuo. El cambio puede ser deliberado o involuntario, para mejorar o para empeorar, correcto o incorrecto, y consciente 0 inconsciente. Para que se considere aprendizaje, este cambio debería ocurrir mediante la experiencia: por la interacción de una persona con su entorno. Los cambios resultantes del aprendizaje influyen en los conocimientos o en la conducta del individuo. Si bien la mayoría de los psicólogos coincidirían con esta afirmación, algunos 
suelen darles mayor importancia a los cambios en los conocimientos, y otros a los cambios en la conducta.

Entre otros muchos entes, la educación formal y específicamente para el tema que nos ocupa; la educación superior busca influir en los cambios de conocimiento y de conducta, diseñando e implementado procesos de enseñanza aprendizaje que garanticen que estos cambios sean permanentes y trasformadores. La evaluación de los aprendizajes que tradicionalmente se le ha asignado la función de dar cuenta de si los cambios esperados ocurren o no, se ha convertido en los últimos tiempos en un proceso innato a los espacios de aprendizaje que cambia su función a una más dinámica y procesal, de manera que no solo le corresponda la valoración del proceso completo, sino de dar cuenta de cómo ocurre ese proceso y cuáles pueden ser las ayudas que el estudiante necesita para alcanzar los cambios propuestos.

En esta nueva propuesta de evaluación como aprendizaje, aparece también una nueva visión de la retroalimentación. Ávila Luna (2009), Amaranti (2010), Osorio y López (2014) se refieren a la retroalimentación como un proceso inherente a los procesos formativos pues de la calidad de la retroalimentación, del manejo del error y de la ayuda justa en el momento justo depende que el aprendizaje se construya de manera más profunda y significativa.

En tal sentido, si retomamos que, en el ámbito educativo, el término retroalimentación, se refiere a la entrega de información al estudiante acerca de su desempeño con el propósito de mejorarlo en el futuro, podemos entender cuánto puede potenciar, la retroalimentación, un proceso de enseñanza aprendizaje más efectivo. Siguiendo a Ávila Luna, (2009) para una persona involucrada en el proceso de enseñanzaaprendizaje, es muy útil conocer durante el transcurso si está logrando los objetivos planteados, al igual que los aspectos que debería mejorar para alcanzarlos con mayor 
facilidad. En este sentido, la retroalimentación le permite al estudiante perfeccionarse y corregirse mientras aprende.

En tal sentido, Amaranti Pesce, (2010) anota que la retroalimentación es central para promover el aprendizaje e involucra a los docentes y estudiantes en un análisis y diálogo sobre el aprendizaje y las metas que deben alcanzar, a partir de lo cual toman decisiones para mejorar dicho proceso. El profesor orienta y proporciona a sus estudiantes estrategias necesarias para mejorar su propio aprendizaje. Significa, como lo menciona Osorio y López, (2014), que la retroalimentación favorece el hecho de que los estudiantes crean en lo que están haciendo y se detengan en un momento a interiorizar sus resultados y así deducir que existen formas adecuadas para realizar las tareas en cada uno de los ámbitos.

Bautista (2011) agrega un elemento fundamental a esta discusión que es el papel del estudiante, pues desde un modelo tradicional es muy habitual que la tarea valorativa del aprendizaje únicamente recaiga en el docente. El autor propone un proceso que implica una notable responsabilidad y actitud pro-activa del estudiante, pues no podemos dejarlo al margen de que asimismo valore si está alcanzando o no su objetivo en la formación y cómo y con qué profundidad los está alcanzando. No obstante, para que dicho proceso de autoevaluación o autorregulación suceda, el acompañamiento y retroalimentación que recibe el estudiante durante su actividad son fundamentales.

Se debe, entonces, también asumir que la retroalimentación debe ser entendida como una actividad dialógica en la que los profesores y estudiantes analizan los resultados de las evaluaciones en comparación con los criterios propuestos y se toman acuerdos de acciones que se pueden desarrollar para mejorar en pos de las metas de aprendizaje. La retroalimentación es central para promover el aprendizaje e involucra a los docentes y estudiantes en un análisis y diálogo sobre el aprendizaje y las metas que deben alcanzar, a partir de lo cual toman decisiones para mejorar dicho proceso. 
Por lo tanto, es de esperar, según Amaranti (2010), que, en un proceso de retroalimentación, el profesor oriente y proporcione a sus estudiantes estrategias necesarias para mejorar su propio aprendizaje. La retroalimentación, agregan Osorio y López (2014) y Berenguer (2010) es parte integral de la evaluación formativa en cuanto ofrece información importante para hacer ajustes en el proceso de enseñanzaaprendizaje con el propósito de que los estudiantes alcancen los objetivos propuestos.

La retroalimentación en el espacio formativo tiene como protagonistas al alumno junto con su docente, la relación se da entre ellos en torno a una tarea particular teniendo como desenlace un impacto, se espera que positivo, sobre el alumno. En primera instancia el docente propone al alumno una situación de aprendizaje, como puede ser el caso de desarrollar algún proyecto, y luego explica los objetivos para dicha tarea exponiendo claramente los criterios fundamentales a tener en cuenta. El alumno debe volcar en tal proyecto todos sus conocimientos, resolver la problemática, y presentarlo al profesor para que lo analice y le brinde una devolución sobre el mismo [sic]. (p. 2)

Por lo anterior, la retroalimentación debe requerir una acción del estudiante, con miras al criterio o a los criterios de evaluación, ya conocidos por él, que lo involucre en su aprendizaje y lo comprometa a avanzar. Puede existir mucha retroalimentación que entregue una información al estudiante sobre el estado de su trabajo, pero no lo oriente con acciones que lo ayuden a mejorar, la retroalimentación debe ser más constructiva. No basta sólo con comentar un trabajo hay que darle un tiempo al estudiante para actuar sobre el comentario. Según Amaranti (2010)

lo que frecuentemente hacemos en la retroalimentación es que hacemos comentarios que ayudan mucho en el trabajo, pero 
después vamos directo al siguiente trabajo, nada se logrará con estos comentarios. Si nosotros dedicamos más tiempo a escribir nuestros comentarios de lo que pasa el estudiante actuando sobre nuestro comentario, no habrá valido la pena. Por lo tanto, como se dijo anteriormente, es necesario darle un tiempo al estudiante para realizar una acción sobre el comentario, ya que la finalidad es mejorar el aprendizaje. (p. 7)

La retroalimentación evaluativa se inscribe según Maldonado (2009) en cinco tipos o clases de acuerdo un contexto de criterios, características y aspectos como los siguientes:

1. Directa: cuando está dirigida a un miembro del grupo en particular.

2. Descriptiva: cuando expresa en forma detallada las características de la conducta a la que está referida.

3. Específica: cuando está referida a una situación determinada en un momento preciso.

4. Inmediata: cuanto está referida a una situación próxima muy cercana.

5. Con expresión emocional: demostrando la emoción y sentimiento que acompaña la comunicación (p. 6).

Estos tipos o clases de retroalimentación no solo no se excluyen entre sí, sino que se complementan y deberían estar presentes en un buen proceso de formativo. Unido a una posible integración de estos tipos de retroalimentación es necesario tener claras, al menos las principales ventajas que estos insumos ofrecen al proceso de aprendizaje. Vives-Varela y Varela-Ruiz (2013) mencionan como ventajas del proceso de retroalimentación adecuada para el aprendizaje los siguientes puntos:

1. Ofrece introspección, que ayuda a los estudiantes a realizar ajustes en su desempeño para mejorarlo.

2. Favorece la autoevaluación al recibir una crítica constructiva. 
3. Aclara las metas y expectativas que se espera sean alcanzadas. Refuerza las acciones y prácticas correctas.

4. Provee las bases para corregir errores.

5. Incrementa la capacidad de incorporar al desempeño la opinión y sugerencias de expertos.

6. Brinda información que no se encuentra en los textos o en los exámenes.

7. Demuestra compromiso e interés del docente en sus aprendices.

8. Promueve una comunicación positiva entre profesores y estudiantes (p.113).

Aunque se han mencionado las ventajas genéricas de una buena retroalimentación, los resultados de esta dependen, según Berenguer (2010), de cada alumno en particular y de su actitud hacia las mismas, la corrección es de esperar que afecte de modo positivo y oriente al alumno de modo estimulante, pues una correcta evaluación motiva al alumno a continuar desarrollando su proyecto a su máximo potencial, por ello es vital la función del docente en esta instancia y su dedicación en obtener una buena retroalimentación. Sin embargo, Shepard (2006), Ibarra, Rodríguez y Gómez (2012) mencionan que la retroalimentación facilita el aprendizaje y que, sin retroalimentación sobre errores conceptuales o retrocesos ineficaces, es probable que el estudiante persista en cometer los mismos errores.

Los procesos de retroalimentación que recibe el estudiante durante su actividad formativa son fundamentales para su aprendizaje. Bautista (2011) indica que la dimensión de la evaluación es muy significativa desde el modelo de profesor que acompaña el aprendizaje, porque desde un modelo tradicional es muy habitual que la tarea valorativa del aprendizaje únicamente recaiga en el docente, pero no así en la propuesta que estamos haciendo. Hemos planteado un proceso que implica una notable responsabilidad y actitud pro-activa del estudiante y no podemos dejarlo al margen de que también valore si está alcanzando o no su objetivo en la formación ( $p$. 62). 


\section{3- Retroalimentación y estrategias cognoscitivas}

A criterio de Lisette Poggioli (1997), existen muchos autores que han definido el término estrategias cognoscitivas. Algunos de ellos son:

1. Las estrategias cognoscitivas son todas las actividades y operaciones mentales en las cuales se involucra el aprendiz durante el proceso de aprendizaje y que tienen por objeto influir el proceso de codificación de la información.

2. Las estrategias hacen referencia a operaciones 0 actividades mentales que facilitan y desarrollan diversos procesos de aprendizaje escolar. Gracias a las estrategias podemos procesar, organizar, retener y recuperar el material informativo que tenemos que aprender, a la vez que planificamos, regulamos y evaluamos esos mismos procesos en función del objetivo previamente trazado o exigido por las demandas de la tarea (p. 52).

Siguiendo la línea de Poggioli (1997), "cuando adquirimos estrategias cognoscitivas, se puede decir que hemos adquirido procedimientos que nos permiten aprender a aprender". Existen diferentes tipos de estrategias cognoscitivas, como por ejemplos, aquellas relacionados con actividades como aprender, recordar, resolver problemas y auto-regularse. La misma autora aclara que la autorregulación se concibe como planificación y el establecimiento de metas y sub-metas con el fin de guiar y probar nuestros procesos, ya sean éstos de memoria, de comprensión, de aprendizaje, de resolución de problemas o de comunicación. Estos procesos se enmarcan dentro de la denominación genérica de metacognición e comprenden dos tipos de actividades:

1) tener consciencia de lo que sabemos o no acerca del material que debemos aprender y de los procesos involucrados en su adquisición; y

2) asumir la regulación de las actividades que debemos realizar para que el aprendizaje sea exitoso (planificar, establecer las demandas de la tarea 
de aprendizaje, atender a la naturaleza de los materiales, monitorear o revisar constantemente el proceso de aprendizaje, evaluar la comprensión.

Por eso, se debe enseñar a los estudiantes a desarrollar sus habilidades metacognitivas, de manera que puedan verbalizar las estrategias utilizadas para cumplir una tarea; si lo hacen, podrán evaluar el proceso realizado. García (2014) afirma que "esta es una instancia para que el profesor retroalimente los intentos metacognitivos de los estudiantes (...), hay que guiarlos para que alcancen su máxima expresión (p. 1)".

La autorregulación, como parte de los procesos de aprendizaje, propicia la integración de los conocimientos a las estructuras cognitivas. Pero, además, integra comportamientos, pensamientos y emociones que permiten la consecución de metas y objetivos académicos mediante el control y la revisión de parte del estudiante. Garello (2013) expone: "Uno de los aspectos más destacados en el monitoreo del desempeño es la retroalimentación, que puede provenir tanto del mismo sujeto, como de los pares o profesores y promueve procesos de reflexión, revisión y optimización de los aprendizajes construidos" (p. 134).

Así las cosas, Cruz (2008) indica que la autorregulación se pone en evidencia cuando en un proceso de aprendizaje, la personas utiliza "diversos procesos de monitoreo activado y de regulación de un número de diversos procesos de aprendizaje". Esto se da:

1. Mediantee las metas que se establecen alcanzar en el acto educaivo.

2. Con las estrategias utilizadas para llegar a esas metas determinadas.

3. Mediante el uso y manejo de recursos que dispone a su alrededor.

4. Aplicando el nivel de esfuerzo requerido.

5. A través de las reacciones como resultado de la retroalimentación.

6. Cuando hay capacidad de autoevaluación (p.23). 
En ese sentido, Amaranti (2010) reafirma que la retroalimentación requiere una acción del estudiante, centrada en el criterio o a los criterios de evaluación, ya conocidos por él, que lo comprometa en su aprendizaje y lo motive a avanzar. Puede existir mucha retroalimentación que entregue una información al estudiante sobre el estado de su trabajo, pero no lo oriente con acciones que lo ayuden a mejorar, la retroalimentación debe ser más constructiva" (p. 4).

\section{4- Condiciones y características de una retroalimentación adecuada}

Como se mencionó anteriormente, la retroalimentación en los procesos de evaluación han existido desde siempre, pero la conciencia de que la retroalimentación debe ofrecerse de manera justa y en el momento oportuno, y qué para que contribuya realmente a un aprendizaje significativo, profundo y transformador la retroalimentación tiene condiciones específicas, no ha sido tan atendida sino hasta que la evaluación se comienza a mirar como un proceso imbricado al proceso de aprendizaje, el cual debe ser formativo y formador y lo más auténtico posible. Vives y Varela (2013), Bossi (2001), Cruz (2008) y Alvarado (2014) aportan elementos importantes para determinar cuáles deben ser estas características y condiciones.

Al tomar en cuenta la definición de realimentación de Vives y Varela (2013), quienes anotan que es una práctica que permite al docente ocupar el lugar de guía, capaz de promover el desarrollo potencial del estudiante y se refiere a ella como "la habilidad que desarrolla el docente al compartir información específica con el estudiante sobre su desempeño, para lograr que el educando alcance su máximo potencial de aprendizaje según su etapa de formación" (p.113), podría decirse que una de las primeras condiciones tiene que ver con la habilidad del docente de destacar los puntos medulares del proceso de aprendizaje para ofrecer la información específica en cada momento del proceso. En ese mismo sentido, Alvarado García (2014) mencionan que 
"para llevar a cabo el proceso de retroalimentación con resultados satisfactorios es importante considerar que son tres las variables que influyen: "las habilidades pedagógicas del tutor, el nivel de actividad de los alumnos y un esfuerzo compartido entre ambas partes" (p. 71).

Bossi (2001) menciona que para llevar a cabo una retroalimentación efectiva hay que seguir los siguientes pasos:

1. Conocer los objetivos del otro y haber observado algunos aspectos, que, en su opinión, ayuden al otro a mejorar su efectividad.

2. Crear un contexto de escucha y aprendizaje. Una conversación de feedback es efectiva si quienes la practican aprenden algo del proceso. Ambos deberían estar abiertos al aprendizaje. Ambos podrían leer y reflexionar, antes de la charla, los enemigos del aprendizaje más comunes.

3. Chequear si el otro te da autoridad para darle feedback en el dominio a tratar. Todo el proceso es inútil si el otro no está dispuesto a escucharte.

4. Especificar sobre qué aspectos o dominios daremos feedback. Deberías armar previamente una matriz que cruce los objetivos que el otro se propuso con las habilidades o dominios que tú crees que el otro debería considerar o mejorar. Luego juntos podrían armar un listado de temas por tratar.

5. Sobre cada punto, invitar al otro a abrir una conversación sinérgica sobre nuevas posibilidades de acción, la cual tendrá más chances de centrarse en lo que queremos producir y escaparnos de las típicas explicaciones del pasado.

6. Para el cierre, es interesante chequear que pasó, durante el mismo, "¿Cómo te sentiste durante la conversación?". Es fundamental que ambas personas se completen; es decir, no dejar "nada en el tintero", que pudiera convertirse en un peso en el futuro para la relación (pp. 2-3). 
Es recomendable llevar a cabo la realimentación durante todo el proceso de enseñanza-aprendizaje, pero de acuerdo con Vives-Varela y Varela-Ruiz (2013) es importante hacerla cuando el estudiante más la necesite, ya que

La realimentación promueve en los novatos de habilidades que progresivamente, le conducen hacia un camino de constante superación y reflexión en el desempeño. Si se ofrece en el momento oportuno, cuando el estudiante está actuando en un contexto determinado, es una herramienta poderosa para el cambio. (p.113)

Para Cruz (2008), una buena retroalimentación:

1. "Ayuda a aclarar lo que es un buen desempeño.

2. Facilita el desarrollo de la reflexión y autoevaluación del aprendizaje.

3. Entrega a los estudiantes información de calidad sobre su aprendizaje.

4. Promueve el diálogo sobre el aprendizaje, con el profesor y los compañeros.

5. Fomenta la autoestima y las creencias motivacionales positivas.

6. Brinda oportunidades para cerrar la brecha entre el desempeño dado y el deseado.

7. Provee información a los profesores, la misma que puede ser utilizada para modificar la enseñanza" (p. 11).

Ahora bien, para que una retroalimentación esté completa según Alvarado (2014), debe incluir tres conceptos:

"Feed-Up", que se refiere a qué dirección está tomando el alumno, si se está dando cuenta hacia dónde va, es básicamente no perder de vista el objetivo de la actividad que se está revisando y hacer referencia, en síntesis, de la actividad previa para ligar el conocimiento previo con el actual; el "Feed Forward" que esencialmente y en retroalimentación enviada por su profesor, debe contestar al alumno las interrogantes sobre 
¿qué sigue ahora? ¿Cómo puede mejorar para la siguiente actividad? Y el "Feed - Back" que le ayuda al alumno a darse cuenta cómo se está desempeñando. (p. 7)

Para Berenguer (2010), una retroalimentación de calidad deberá ser: cualitativa, cuantitativa y oportuna. Alvarado (2014) aporta que hay tres factores que hay que tener en cuenta para que se dé una buena retroalimentación, en primer lugar el clima del especio de aprendizaje, en segundo lugar la confianza en el trato, y en tercero la posibilidad de que se dé un buen diálogo entre docente y alumno. Berenguer (2010) también apunta: "estos factores ayudan al alumno a que se sienta seguro, pudiendo reflexionar tanto como debatir o aclarar cuestiones no comprendidas, y que al momento de la devolución pueda reconocer sus errores, modificarlos, incorporando las pautas recibidas de manera constructiva" (p. 4).

\section{5- Entornos virtuales y retroalimentación}

Las transformaciones que se han dado con la incorporación de las tecnologías de la información y comunicación, no pueden dejar de lado el campo de la evaluación y algunos ya se refieren a estos procesos como e-evaluación, orientada al e-aprendizaje. Este se entiende, según Rodríguez e Ibarra, (2011) como "el proceso de aprendizaje, mediado por medios tecnológicos, a través del cual se promueve y potencia el desarrollo de competencias útiles y valiosas para el presente académico y el futuro laboral de los estudiantes universitarios como profesionales estratégicos" (p. 37). Supone realizar la evaluación sobre tareas de aprendizaje, ofrecer una retroalimentación positiva, la implicación del estudiante en su proceso de evaluación, y poder utilizar instrumentos tecnológicos para llevarla a cabo.

Las facilidades de retroalimentación que los entornos virtuales de aprendizaje ofrecen, permiten la identificación de aspectos mejorables en los materiales y en la acción docente, y facilita, por tanto, un proceso de mejora continua. Además, los entornos 
virtuales según Esteban (2000) y Mestre, Fonseca y Valdés (2007) permiten avanzar en la calidad, facilidad y productividad de las interacciones socio cognitivas, que incluyen la retroalimentación.

Continuando con los entornos virtuales, García-Beltrán, Martínez y Tapia (2006), agregan que la utilización de un entorno virtual proporciona una respuesta inmediata (retroalimentación) de los resultados de los ejercicios o procesos de evaluación. La ventaja de la retroalimentación inmediata constituye una clave fundamental en el proceso de aprendizaje, al ejercer como elemento de constante motivación al esfuerzo del alumno y como herramienta para la orientación eficaz en sus actividades. Los autores mencionados completan esta idea, puntualizando que el trinomio interacción, retroalimentación y atención personalizada proporcionan el establecimiento o fomento de relaciones personales y cognitivas entre los participantes de un curso en un medio virtual.

Villar y Alegre (2013), expresan que las transformaciones que proporcionan las TIC también deberán darse en el componente de la evaluación, y establecer en este caso, niveles de diferencia en lo que respecta a su aplicación en los contextos de e-learning 1.0 y 2.0, ya que desgraciadamente muchas veces se cambian las metodologías, las formas de ofrecer y diseñar los contenidos, las estrategias didácticas y las herramientas de comunicación que se utilizan con los estudiantes. Pero se siguen movilizando estrategias de evaluación de una formación bancaria y tradicional, en vez de potenciar el desarrollo de una evaluación auténtica; que se refiere según estos mismos autores "a los procesos y productos en los que se implica un estudiante porque son importantes para la vida más allá de la escuela o universidad" ( p. 11).

Un estudiante en un entorno en línea espera que la interacción con el profesor sea fluida y sistemática. Por tal razón, la sistematización en la atención al estudiante, a sus dudas y sus necesidades de orientación para el aprendizaje debe formar también parte 
fundamental dentro del conjunto de estrategias docentes en un entorno virtual. La situación contraria y muy negativa al estudiante acompañado, sin duda, es el estudiante abandonado.

Por ello, la retroalimentación inmediata es fundamental. El estudiante necesita saber si progresa de forma adecuada, precisa sentir que sus dudas tienen respuestas inmediatas y tiene que saber que el profesor está haciendo un seguimiento constante de sus actividades. La tutorización y el seguimiento en un entorno virtual es fundamental y requiere una formación específica. Silva (2011) puntualiza que hay que diseñar y moderar los entornos virtuales teniendo en cuenta el origen social y cultural de los estudiantes, los tipos de contribuciones que deben realizar, planificar su frecuencia, los espacios más adecuados, las herramientas más apropiadas, etc.

Sobrino (2011), Gros (2012) y Cabero y Barroso (2013) hacen alusión a la web 2.0 por la potencialidades en cuanto a personalización, apertura, interactividad, ubicuidad, inmediatez, flexibilidad, interoperabilidad y colaboración que ofrecen en los entornos virtuales de aprendizaje, los cuales son elementos claves para promover, entre muchos otros aspectos, la retroalimentación en el marco de la evaluación para aprender, pues ofrecen una serie de ventajas como: crear interdependencia positiva entre los miembros, generar debates en torno a la búsqueda de estrategias de uso y resolución de problemas, facilitar el intercambio de información y la construcción social del conocimiento.

\section{Marco metodológico}

Este estudio se realizó mediante la revisión bibliográfica, cuya recolección de datos se llevó a cabo en mayo y junio del 2015. Para hacerlo, se recurrió a fuentes provenientes de internet como google académico, e-biblioteca de la UNED de los cuales se recurrió a artículos especializados y científicos relacionados con el tema de realimentación. 
Primero, se llevó a cabo un listado de autores relacionados con el tema central. Luego de realizar una lectura rápida de los resúmenes e índices; algunos autores se descartaron; de otros textos, sí se seleccionaron citas que explicaran el concepto de realimentación y la relación con el proceso educativo, y se elaboraron fichas. Estas fichas contienen citas directas o indirectas, además de la referencia de cada una.

Posteriormente, se analizó el contenido y se encontraron cuatro categorías:

- Realimentación y evaluación de los aprendizajes

- Realimentación y aprendizaje

- Realimentación y estrategia cognoscitivas

- Realimentación en entornos virtuales de aprendizaje

A partir de estas líneas temáticas, se extrajeron evidencias sobre las características que debe presentar la realimentación. De ese modo, en el marco teórico se presentan fundamentos que permiten comprender y contextualizar las características de tal proceso cuando es parte de la estrategia evaluativa que se lleva a cabo en el marco del proceso educativo formal implementado en un entorno virtual.

\section{IV- Discusión}

Para Álvarez (2001), la evaluación de los aprendizajes es un concepto polisémica y controversial. Apunta que "todos hablamos de evaluación, pero cada uno conceptualiza e interpreta este término con significados distintos". (p. 11). La evaluación de los aprendizajes tiende a confundirse con medir o con examinar y a ser asumida como un proceso final que da un informe de lo aprendido en un momento dado, el cual es traducido, la mayoría de las veces, a números.

La polisemia y la controversia aparecen cuando el concepto de evaluación, en la mayoría de las ocasiones, se impone a la práctica según las necesidades y las 
funciones que esta debe cumplir. Además, es posible que cuando no se tenga claro a cuál enfoque de evaluación de los aprendizajes se quiere atender no se encuentre una definición clara o convincente de este término. Empero, si nos ubicamos en un enfoque alternativo, el cual le da un lugar preponderante a la formación, no será tan difícil asumir una definición, pues el carácter formativo y formador de esta nos ubica en una evaluación alternativa al servicio del aprendizaje.

Tratando, entonces, de llegar a un concepto que nos acerque a un enfoque alternativo se puede decir que la evaluación de los aprendizajes significa valorar el aprendizaje de los estudiantes durante un proceso formativo. Se refiere al proceso sistemático, integral y continuo mediante el cual se determina el grado en que se están logrando los objetivos del aprendizaje (conocimientos, habilidades, destrezas, valores, actitudes, procedimientos $u$ otro aspecto evaluable) para realizar los ajustes necesarios que mejoren o corrijan las debilidades del proceso de aprendizaje.

El concepto anterior supera la evaluación como la mera medición, por ejemplo, al medir se puede determinar que una mesa tiene $1 \mathrm{~m}$ de largo y $50 \mathrm{~cm}$ de ancho, pero, mediante la evaluación se puede indicar cuán larga o corta resulta para las personas que van a usarla, si la mesa cabe en el espacio que se destinó para su colocación, si se deben realizar ajustes al contexto para colocarla, si se debe buscar una mesa con otras proporciones y si las necesidades de las personas son las correctas.

Por lo tanto, la información que se obtiene de la medición no necesariamente refleja cómo el estudiante alcanzó los objetivos de aprendizaje establecidos ni cómo el profesor tutor realizó su tarea docente para propiciar el alcance los objetivos de aprendizaje. En razón de ello, evaluar puede relacionarse con términos como: aplicación de exámenes, memorización de contenidos, control, función del profesor, evaluación de resultados y por supuesto, con medición. 
Otro hecho que se impone en la práctica evaluativa es la examinación del error para excluir; es decir, manejarlo como criterio negativo o débil y no como una nueva oportunidad para aprender; o, peor aún, no tomar en cuenta los puntos positivos, los aciertos o serendipias como espacios para hacer que el estudiante vaya más allá de lo esperado.

Al asumir un concepto de evaluación intrínseco al proceso de aprendizaje, que, entre otras cosas, utiliza la medición pero la supera ampliamente y que encuentra o ayuda a encontrar errores, vacíos o aciertos para superarlos, entonces debe aprovecharse su arma más poderosa: la retroalimentación.

La retroalimentación, entendida como un espacio de comunicación horizontal en donde docentes-estudiantes y estudiantes-estudiantes interactúan con el objeto de estudio y el contexto para alcanzar un cambio cognitivo y conductual, debe abrir espacios extensos y profundos a la pregunta tipo socrática, a los retos, a las sugerencias, a las oportunidades, al razonamiento y a la construcción colaborativa de nuevos aprendizajes.

Visto de esta manera, la mayoría de los autores citados en este estudio, Amaranti Pesce (2010), Lozano y Tamez (2014), Román Maldonado (2009), Osorio y López (2014) Vives-Varela y Varela-Ruiz (2013) y Ávila Luna (2009) concuerdan en:

1. La acción de retroalimentar el proceso de aprendizaje no puede dejarse para el final, sino el mismo debe ser sistemático y continuado durante todo el proceso.

2. La tarea de retroalimentar no es exclusiva del docente; por el contario, es necesaria la participación de los pares que coevalúan y del mismo estudiante que autoevalúa y autorregula su propio proceso. 
3. Promover el aprendizaje e involucrar a los participantes del proceso educativo a través del análisis y el diálogo que retroalimenta el aprendizaje y las metas que deben alcanzar es una labor imprescindible de la evaluación formativa y formadora.

4. El compromiso con una retroalimentación que promueve el aprendizaje profundo debe partir de una planificación cuidadosa que desde los objetivos deja ver los alcances que tendrá un proyecto (curso-módulo, unidad) específico, para que en un proceso dilógico y reflexivo, docente y estudiantes logren lo propuesto. Una planificación adecuada permite tener claro los hitos en donde es más necesaria la retroalimentación, la forma en que se hará y los espacios de superación que tendrá el estudiante.

\section{Conclusiones}

Nuestro modelo pedagógico UNED (2004) propone que para avanzar hacia una evaluación de tipo formativo, es necesario:

1. Ofrecer una pauta de las actividades que se han de realizar y sugerir un ritmo de trabajo concreto que, según la experiencia, garantice mejor el logro de los objetivos en el tiempo disponible.

2. Que cada actividad que el estudiante realice se debe convertir, por una parte, en una oportunidad para asegurar su participación activa en la construcción del propio conocimiento; y, por otra parte, en una ocasión para facilitar la guía necesaria del docente sobre cómo orientar y enfocar el estudio.

3. Apuntar claramente a un proceso consciente y sistemático de retroalimentación proponiendo la autorregulación y la coevaluación como elementos esenciales del proceso de evaluación de los aprendizajes. 
Con el mismo espíritu del modelo pedagógico de la UNED, el plan académico 20122017 apunta hacia una evaluación de aprendizajes de calidad, formativa y formadora, que apueste por aprendizajes significativos, que se aplique a nuevos contextos, que contemple el perfil y particularidades de los estudiantes que atiende y que no sea solamente un elemento de medición y reproducción del conocimiento, sino una forma de aprendizaje.

La literatura sobre fundamentos teóricos y lineamientos prácticos en el campo de la evaluación de los aprendizajes en general y de manera específica sobre la evaluación alternativa, formativa, formadora, auténtica que promueve el aprendizaje significativa es robusta, innovadora y propositiva.

La UNED cuenta con equipos de apoyo con una alta solvencia académica y equipos docentes preocupados y conscientes por un cambio en la evaluación de los aprendizajes y un equipamiento tecnológico en contante mejora.

Lo anterior significa que tenemos todas las potencialidades necesarias para implementar de una vez por todas estrategias de aprendizaje y de evaluación, a nivel institucional y de cátedras, que estén acorde con nuestro modelo de educación a distancia, a nuestro contexto. Pero, sobre todo, a lo que aspiramos: procesos de enseñanza-aprendizaje que permitan la construcción de aprendizajes complejos y trasformadores y no hay duda de que la retroalimentación, propuesta oficialmente en nuestro modelo desde el 2004, es un elemento medular sin el cual es difícil alcanzar lo propuesto. 
Características de la retroalimentación como parte de la estrategia evaluativa durante el proceso de enseñanza aprendizaje en entornos virtuales: una perspectiva teórica

Julia Pérez Chaverri, Marianela Salas Soto

\section{Referencias}

Alvarado, M. A. (2014). Retroalimentación en educación en línea: una estrategia para la construcción del conocimiento. RIED, 17(2), 59-73. Recuperado de http://ried.utpl.edu.ec/sites/default/files/files/file/archivo/volumen172/retroalimentacion.pdf

Amaranti, M. (2010). Evaluación de la educación: Concepciones y prácticas de retroalimentación de los profesores de lenguaje y comunicación de primer año de educación media, investigación cualitativa con estudio de caso [Archivo PDF tomado de una página web]. Recuperado de http://www.adeepra.org.ar/congresos/Congreso\%20IBEROAMERICANO/EVAL UACION/RLE2488_Amaranti.pdf

Arancibia, V. (1997). Los sistemas de medición y evaluación de la calidad de la educación. OREALC-UNESCO. Santiago de Chile. Recuperado de http://unesdoc.unesco.org/images/0018/001836/183651s.pdf

Ávila, P. (2009). La importancia de la retroalimentación en los procesos de evaluación [Archivo PDF tomado de una página web]. Recuperado de http://clasev.net/v2/pluginfile.php/56479/mod_resource/content/1/LA\%20RETR OALIMENTACION\%20Extracto\%20del\%20documento.pdf

Barberá, E. (2006). Aportaciones de la tecnología a la e-Evaluación [Archivo PDF tomado de una página web]. Recuperado de http://www.um.es/ead/red/M6/barbera.pdf

Berenguer, S. (2010). Una correcta evaluación [Archivo PDF tomado de una página web]. Recuperado de http://fido.palermo.edu/servicios_dyc/blog/images/trabajos/13409_54196.pdf

Bordas, I. y Cabrera, F. (2001). Estrategias de evaluación de los aprendizajes centrados en el proceso. Revista Española de Pedagogía. Recuperado de http://cmapspublic3.inmc.us/rid=1GLSW84JS-WYZWX0$\mathrm{H} 40 /$ Evaluaci\%C3\%83\%C6\%92\%C3\%82\%C2\%B3n\%20del\%20Proceso\%20d e\%20Aprendizaje.pdf

Bossi, I. (2001). ¿Para qué dar Feedback?: ¿Cómo hacerlo en forma efectiva? [Archivo PDF tomado de una página web]. Recuperado de http://www.ucema.edu.ar/u/jib/Documentos/DT_05_-

_El_feedback_efectivo_v2.pdf 
Barroso, J. y Cabero, J. (2013). Replanteando el e-learning: hacia el elearning 2.0 Campus Virtuales, II(02), 2013, Revista Científica de Tecnología Educativa; ISSN: 2255-1514.

Bautista, G. (2011). El acompañamiento del estudiante: profesorado para una nueva forma de aprender. En Gros, B. (Ed.). Evolución y retos de la educación virtual.

Coll, C., Mauri, T. y Rochera, M. J. (2012). La práctica de evaluación como contexto para aprender a ser un aprendiz competente. Profesorado: Revista de currículum y formación del profesorado, 16(1), 49-59. Recuperado de http://www.ugr.es/ recfpro/rev161ART4.pdf

Cruz, M. L. (2008). Evaluación formativa y autorregulación: Un estudio de caso [Archivo PDF tomado de una página web]. Recuperado de http://repositorio.usfq.edu.ec/bitstream/23000/254/1/88004.pdf

Durán, Y. y Valerio, C. (2008). Evaluación alternativa en los entornos virtuales [Archivo PDF tomado de una página web]. Recuperado de http://observatoriotecedu.uned.ac.cr/media/evaluacion_alternativa.pdf

Esteban, M. (2000). El diseño de entornos de aprendizaje constructivista [Archivo PDF tomado de una página web]. Recuperado de http://www.um.es/ead/red/6/documento6.pdf

Fernández, A. (2005). La evaluación de los aprendizajes en la universidad: Nuevos enfoques [Archivo PDF tomado de una página web]. Recuperado de http://web.ua.es/es/ice/documentos/recursos/materiales/ev-aprendizajes.pdf

Fernández, I., García, M. A. y González, P. (2004). Una experiencia de evaluación alternativa en las escuelas técnicas del campus de Gijón (Universidad de Oviedo) [Archivo PDF tomado de una página web]. Recuperado de http://www.upc.edu/euetib/xiicuieet/comunicaciones/din/comunicacions/272.pdf

García, C. (2014). Metacognición, aprendizaje y talento académico [Archivo PDF tomado de una página web]. Recuperado de http://www.pentauc.cl/wp/wpcontent/uploads/2014/04/500-palabra-ECG.pdf

García-Beltrán, A., Martínez, R., Jáen, J. A. y Tapia, S. (2006). La autoevaluación como actividad docente en entornos virtuales de aprendizaje/enseñanza [Archivo PDF tomado de una página web]. Recuperado de http://www.um.es/ead/red/M6/garcia_beltran.pdf 
Gros, B. (ed.). (2011). Evolución y redes de la educación virtual: Construyendo el eLearning del siglo 21. Barcelona, España: Editorial UOC.

Ibarra, M. S., Rodríguez, G. y Gómez, M. A. (2012). La evaluación entre iguales: beneficios y estrategias para su práctica en la universidad. Revista de Educación, 359, 206-231. Recuperado de http://www.mecd.gob.es/dctm/revista-deeducacion/articulosre359/re35911.pdf?documentld=0901e72b813d72cf

Jiménez, F. (2015). Uso del Feedback como estrategia de la evaluación. Aportes desde un enfoque socioconstruccionista. Revista Electrónica Actualidades Investigativas de la Educación. Recuperado de http://www.redalyc.org/pdf

Lozano, F. G. y Tamez, L. A. (2014). Retroalimentación formativa para estudiantes de educación a distancia. Revista Iberoamericana de Educación a Distancia, 2, 197-221.

Luzardo, H. (s.f.). La evaluación tradicional en el enfoque tecnológico. Revista de Tecnología de Información y Comunicación en Educación, 3(2). Recuperado de http://servicio.bc.uc.edu.ve/educacion/eduweb/Vol3n2/art5.pdf

Maldonado, R. (2009). Sobre la retroalimentación o feedback en la educación superior online. Revista Virtual Universidad Católica del Norte, 26, 1-18. Recuperado de http://www.redalyc.org/pdf/1942/194215516009.pdf

Mestre, U., Fonseca, J. J. y Valdés, P. R. (2007). Entornos virtuales de enseñanzaaprendizaje. La Habana, Cuba: Editorial Universitaria.

Ministerio de Educación de Guatemala (2010). El currículo organizado en competencias: Evaluación de los aprendizajes. Guatemala: MINEDUC.

Osorio, K. y López, A. (2014). La retroalimentación formativa en el proceso de enseñanza-aprendizaje de estudiantes en edad preescolar. Revista Iberoamericana de Evaluación Educativa, 7(1), 13-30. Recuperado de http://www.rinace.net/riee/numeros/vol7-num1/art1.pdf

Pogglioli, L. (1997). Estrategias cognoscitivas: una perspectiva teórica. Recuperado de http://braulioedunet.webcindario.com/estrategias\%20cognositivas.pdf

Ramírez, L. A. y Cañizales, K. I. (2013). Entornos virtuales de aprendizaje (EVA): aproximación al mejoramiento de la calidad comunicacional en una universidad venezolana. Associaçao Brassileira de Eduçao a Distância, 12, 397-422. Recuperado de 
http://www.abed.org.br/revistacientifica/_Spanish/2013/8A_Artigo_Rbaad_Esp anhol_2ed.pdf

Sans, A. (2008). La evaluación de los aprendizajes: Construcción de instrumentos. Barcelona, España: Editorial Octaedro.

Shepard, L. (2006). La evaluación en el aula [Archivo PDF tomado de una página web]. Recuperado de http://www.tec.ac.cr/sitios/Docencia/ceda/Boletin_CEDA/PDF_s/aprendizaje_e n_el_aula.pdf

Silva Quiroz, J. (2011). Diseño y moderación de entornos virtuales de aprendizaje (EVA). Santiago, Chile: Editorial UOC.

Sobrino, Á. (2011). Proceso de enseñanza-aprendizaje y web 2.0: valoración del conectivismo como teoría de aprendizaje post-constructivista. Revista Estudios sobre Educación, 20,117-140. Recuperado de http://dadun.unav.edu/bitstream/10171/18344/2/ESE\%20117-139.pdf

Sotomayor, G. (2010). Las redes sociales como entornos de aprendizaje colaborativo mediado parta segundas lenguas. Revista Electrónica de Tecnología Educativa, 34, 1-16. Recuperado de http://edutec.rediris.es/Revelec2/Revelec34/pdf/Edutec-e_n34_Sotomayor.pdf

Universidad Estatal a Distancia (UNED). (2004). Modelo pedagógico de la UNED. Recuperado de http://goo.gl/7fl74w

Villar, L.M y Alegre, O. M. (2013). Los portafolios electrónicos en el hemisferio de la Evaluación auténtica. Madrid: Síntesis.

Vives-Varela, T. y Varela-Ruiz, M. (2013). Realimentación efectiva. Investigación en Educación Médica, 2(6), 112-114. Recuperado de http://riem.facmed.unam.mx/sites/all/archivos/V2Num02/08_PEM_REALIMEN TACION_EFECTIVA.pdf

Woolfolk, A. (2010). Psicología Educativa (10ª ed.). México: Pearson Educación. 